\title{
A checklist of avian fauna at Jeypore Reserve Forest, eastern Assam, India with special reference to globally threatened and endemic species in the Eastern Himalayan biodiversity hotspot
}

\author{
Prasanta Kumar Saikia ${ }^{1} \&$ Oinam Sunanda Devi ${ }^{2}$ \\ ${ }^{1}$ Associate Professor, ${ }^{2}$ Research Scholar, \\ Animal Ecology and Wildlife Biology Lab, Department of Zoology, Gauhati University, Guwahati, Assam 781014, India \\ Email: ${ }^{1}$ saikiapk@rediffmail.com (corresponding author), ${ }^{2}$ sunan_o@rediffmail.com
}

\begin{abstract}
Avifaunal survey of an Eastern Himalayan biodiversity hotspot area - Jeypore Reserve Forest was carried out from July 2007 to July 2009. Altogether 270 species of avian fauna belonging to 56 families were identified during the survey, of which 58 species were frugivorous, 34 omnivorous, 15 carnivorous, 22 picivorous, five grainivorous and 136 were insectivorous in nature. Of all the bird species recorded, 14 were globally threatened species, five were endemic to this region, 153 were resident common species, 87 resident rare species, 16 migratory common species and 14 were migratory rare species. The endemic species recorded in the study area were Anorrhinus tickelli, Sphenocichla humei, Pellorneum palustre, Yuhina bakeri and Heterophasia pulchella. The globally threatened species include two Critically Endangered, Gyps indicus and Gyps bengalensis, one Endangered, Cairina scutulata, four Vulnerable, Leptoptilos javanicus, Francolinus gularis, Columba punicea, Turdoides longitostris, and seven Near Threatened, Anhinga melanogaster, Arborophila atrogularis, Anorrhinus tickelli, Buceros bicornis, Sphenocichla humei, Pellorneum palustre and Ichthyophaga ichthyaetus.
\end{abstract}

Keywords: Biodiversity hotspot, birds, Eastern Himalaya, endemic, globally threatened.

Date of publication (online): 26 April 2011

Date of publication (print): 26 April 2011

ISSN 0974-7907 (online) | 0974-7893 (print)

Editor: Dipankar Ghose

Manuscript details:

Ms \# 02340

Received 05 November 2009

Final received 04 February 2011

Finally accepted 20 March 2011

Citation: Saikia, P.K. \& O.S. Devi (2011). A checklist of avian fauna a Jeypore Reserve Forest, eastern Assam, India with special reference to globally threatened and endemic species in the Eastern Himalayan biodiversity hotspot. Journal of Threatened Taxa 3(4): 1711-1718.

Copyright: Prasanta Kumar Saikia \& Oinam Sunanda Devi 2011. Creative Commons Attribution 3.0 Unported License. JoTT allows unrestricted use of this article in any medium for non-profit purposes, reproduction and distribution by providing adequate credit to the authors and the source of publication.

Acknowledgements: The authors acknowledge the Department of Science and Technology, Government of India, for financial support to conduct the field survey of avian fauna in north and south bank landscapes of Eastern Himalayan biodiversity Hotspot. The authors are also thankfu to the Ministry of Environment and Forests Government of Assam for providing permission to conduct the survey work.

\section{OPEN ACCESS | FREE DOWNLOAD (C) (i) @}

The Jeypore Reserve Forest is one among the few remaining tropical forest patches of eastern Assam which is a part of the Eastern Himalaya biodiversity hotspot region. This reserve is relatively undisturbed in terms of intrusions and disruptions by humans compared to other protected areas of the state. Jeypore along with five other protected areas form the Upper Dihing West Complex, an Important Bird Area (IBA) of eastern Assam, IBA Site No. IN-AS-45 (Islam \& Rahmani 2004). Together they form the largest contiguous tropical rainforest area extent in the whole Brahmaputra Valley (Choudhury 1996). These sites are famous for the largest known population of the Endangered White-winged Duck Cairina scutulata (Choudhury 1996, 1998). Apart from this, the area also harbours five species of hornbills including the rarer Brown Hornbill Anorrhinus tickelli and the Rufousnecked Hornbill Aceros nipalensis (Choudhury 2000). Due to its unique habitat and terrain, the site harbours a large diversity of avian fauna including endemic and globally threatened species. Past studies on avifauna of the reserve forest are restricted to a few personal comments and observations but detailed avifaunal surveys were not done specifically. Data on avian fauna of the reserve is deficient even though it is a part of an important IBA of eastern Assam which provides refuge to a large number of endemic and threatened species. Detailed ecological studies of the endemic and globally threatened species are required for their conservation. Therefore, the present study was conducted to provide up to date information about the avian diversity of the reserve forest and their habitat utilization patterns in order to provide baseline information for future conservation programmes. 


\section{Study Area}

The Jeypore Reserve Forest (JRF) is located at Dibrugarh District of eastern Assam which falls between $27^{\circ} 06^{\prime}-27^{\circ} 16^{\prime} \mathrm{N}$ and $95^{\circ} 21^{\prime}-95^{\circ} 29^{\prime} \mathrm{E}$ (Fig. 1). The total area of the JRF is $108 \mathrm{~km}^{2}$. The terrain of the area varies with slightly undulating plains to hills which are the foothills of the Patkai Range. The JRF is continuous with the forests of Arunachal Pradesh. Burhi-Dihing and the Dilli rivers form a part of the boundary of the reserve. Many small perennial streams and nullahs also flow within the forest. Swamps and grassland patches also occur inside the forest (Kakati 2004). This forest is a part of an important IBA-the Upper Dihing West Complex, IBA Site No. IN-AS-45 and was notified as a reserve forest way back in 1888 (Kakati 2004). The habitat is tropical rainforest, Champion \& Seth (1968) described it as "Assam Valley tropical wet evergreen forest" (category 1B/C1) also called the upper Assam Dipterocarpus - Mesua forest. The forest is characterized by a top canopy dominated by Dipterocarpus macrocarpus reaching heights of $50 \mathrm{~m}$, a middle canopy dominated by Mesua ferrea and Vatica lanceaefolia and undergrowth consisting of woody shrubs such as Saprosma ternatum, Livistonia jenkinsiana and canes Calamus erectus, etc., (Kakati 2004). Bamboo species such as Dendrocalamus hamiltonii, Pseudostachyum polymorphum and climbers such as Derris oblonga are common. The major fauna of the reserve include large mammals such as Elephant Elephas maximus, Barking Deer Muntiacus muntjak, Bengal Tiger Panthera tigris, Leopard Panthera pardus, Clouded Leopard Neofelis nebulosa, Wild Boar Sus scrofa, etc. Primates such as Hoolock Gibbon Hoolock hoolock, Capped Langur Trachypithecus pileatus and Rhesus Macaque Macaca mulatta are also found in the reserve. Rock Python Python molurus and Banded Krait Bungarus fasciatus are some notable reptilian species. Besides these major faunas the reserve also harbours a large diversity of butterflies and arachnid species. Although the forest is located in a matrix of tea plantations, settled agriculture and rural settlements, the degree of disturbance is much less compared to other protected areas of the state. The peripheral areas of the forest are encroached by the

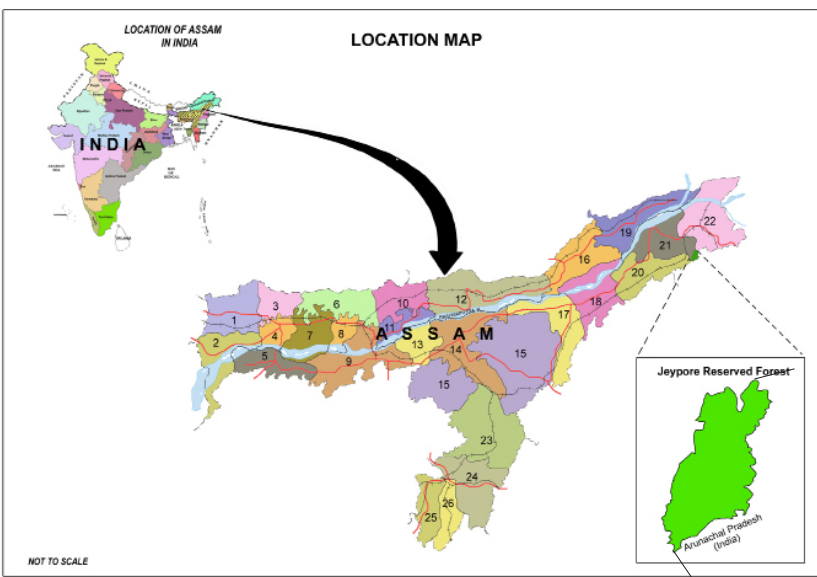

Figure 1. Map showing the study area of Jeypore Reserve Forest in eastern Assam.




local people for tea plantations but the core area of the forest is intact and without any disturbance.

\section{Methods}

Field surveys were conducted for a period of two years starting from July 2007 up to July 2009 covering all the seasons i.e. Summer (March-June), Monsoon (July-October) and Winter (NovemberFebruary). The surveys followed line transect and point count methods (Bibby et al. 1992). Altogether 16 permanent line transects of $2 \mathrm{~km}$ length and $50 \mathrm{~m}$ breadth each were laid randomly, four transects each on all the major habitats of the forest closed canopy, grassland, open canopy and degraded areas near the forest edge. Also point counts were conducted along the line transects of each habitat on different days. Points were of $50 \mathrm{~m}$ radius, at least $100 \mathrm{~m}$ apart from each other to avoid overlap and counts were of $5 \mathrm{~min}$ duration. In each habitat, 15 point count surveys were carried out. All the counts were carried out early morning, during the first three hours after sunrise as counts need to be carried out at the time of highest bird activity (Buckland et al. 1993). Opportunistic observations were also added to the list so as not to miss any species during the survey period. Feeding guilds were classified as per direct observations and available literatures Ali \& Ripley (1987). Birds were identified using field guide books of Ali \& Ripley (1987) and Grimmett et al. (2000). The common and scientific names of the birds given in the checklist followed the Birds of the World, recommended English names (Gill \& Wright 2006). The threatened status of the birds given in the checklist is as per IUCN Red List of Threatened Species (Birdlife International 2001a,b). The common-rare, resident-migratory status of the birds are classified as per Saikia \& Saikia (2000).

$\mathrm{C}$ - Common species which are encountered frequently (eight times during 10 survey trips).

$\mathrm{R}$ - Rare species which are encountered less frequently (1-2 times during 10 survey trips).

$\mathrm{R}$ - Residents, species found in the study area throughout the year.

WM - Winter Migrants, species found in the study area only during the winter.

SM - Summer Migrants, species visiting the area during the summer seasons.

$\mathrm{Vr}$ - Vagrants, species that are not regular, winter migrants or breeding migrants and hence a stray bird sighting.

\section{Results}

A total of 270 species of birds belonging to 56 families were identified during the survey (Table 1). Of these, 58 species were frugivorous, 34 omnivorous, 15 carnivorous, 22 piscivorous, five granivorous and 136 were insectivorous in their feeding guild. Out of 270 species of birds, 14 were globally threatened species and five endemic species including 30 migratory species, of which 25 were winter migrants, five summer migrants and one vagrant species. Family Timaliidae dominates the list with 35 species belonging to 20 genera followed by family Muscicapidae with 20 species under 14 genera and family Columbidae followed close by with 15 species belonging to six genera. Nine species out of these 270 are new records of the area belonging to the families, viz., Nectariniidae (3), Dicacidae (1), Sittidae (1), Muscicapidae (1), Pittidae (1), Psittacidae (1) and Charadridae (1). Out of the 270 species, 153 are resident common species, 87 are resident rare species, 16 are migratory common species and 14 are migratory rare species. Brown Hornbill Anorrhinus tickelli, Wedge-billed Wren-Babbler Sphenocichla humei, Marsh Babbler Pellorneum palustre, White-naped Yuhina Yuhina bakeri and Beautiful Sibia Heterophasia pulchella are the five endemic species. The 14 globally threatened species include two Critically Endangered species, Long-billed Vulture Gyps indicus and Indian Whitebacked Vulture Gyps bengalensis, one Endangered species, White-winged Duck Cairina scutulata, four Vulnerable species, Lesser Adjutant Stork Leptoptilos javanicus, Swamp Francolin Francolinus gularis, Purple Wood Pigeon Columba punicea, Slenderbilled Babbler Turdoides longitostris and seven Near Threatened species viz. Oriental Darter Anhinga melanogaster, White-cheeked Partridge Arborophila atrogularis, Brown Hornbill Anorrhinus tickelli, Great Pied Hornbill Buceros bicornis, Wedge-billed Wren-Babbler Sphenocichla humei, Marsh Babbler Pellorneum palustre and Greater Grey-headed FishEagle Ichthyophaga ichthyaetus.

\section{Discussion}

Eastern Assam falls under the endemic bird area of Eastern Himalaya which is one of the most important 
Table1. Checklist of birds of Jeypore Reserve Forest, identified during the two year study period starting from July 2007 up to July 2009.

\begin{tabular}{|c|c|c|c|}
\hline & Common name & Scientific name & Status \\
\hline & Phasianidae & & \\
\hline 1 & Red Jungle Fowl & Gallus gallus & $\mathrm{R}, \mathrm{C}$ \\
\hline 2 & $\begin{array}{l}\text { White-cheeked } \\
\text { Partridge }\end{array}$ & Arborophila atrogularis & $\begin{array}{l}\mathrm{R}, \mathrm{r} \\
\text { (NT) }\end{array}$ \\
\hline 3 & $\begin{array}{l}\text { Rufous-throated } \\
\text { Partridge }\end{array}$ & Arborophila rufogularis & $\mathrm{R}, \mathrm{r}$ \\
\hline 4 & Kaleej Pheasant & Lophura leucomelanos & $R, r$ \\
\hline 5 & $\begin{array}{l}\text { Grey Peacock } \\
\text { Pheasant }\end{array}$ & Polyplectron bicalcaratum & $R, r$ \\
\hline 6 & Barred Buttonquail & Turnix suscitator & $\mathrm{R}, \mathrm{C}$ \\
\hline 7 & Small Buttonquail & Turnix sylvatica & $\mathrm{R}, \mathrm{C}$ \\
\hline 8 & Blue-breasted Quail & Coturnix chinensis & $R, r$ \\
\hline 9 & Black Francolin & Francolinus francolinus & $\mathrm{R}, \mathrm{C}$ \\
\hline \multirow[t]{2}{*}{10} & Swamp Francolin & Francolinus gularis & $\begin{array}{l}\text { R, r, } \\
\text { VU }\end{array}$ \\
\hline & Anatidae & & \\
\hline 11 & Ruddy Shellduck & Tadorna ferruginea & WM, C \\
\hline 12 & White-wing Wood Duck & Cairina scutulata & $\begin{array}{l}\mathrm{R}, \mathrm{r} \\
(\mathrm{EN})\end{array}$ \\
\hline \multirow[t]{2}{*}{13} & Lesser Whistling Teal & Dendrocygna javanica & $\mathrm{R}, \mathrm{C}$ \\
\hline & Ciconnidae & & \\
\hline 14 & Openbill stork & Anastomus oscitans & $\mathrm{R}, \mathrm{C}$ \\
\hline \multirow[t]{2}{*}{15} & Lesser Adjutant Stork & Leptoptilos javanicus & $\begin{array}{l}\mathrm{R}, \mathrm{C} \\
\text { (VU) }\end{array}$ \\
\hline & Ardeidae & & \\
\hline 16 & Cattle Egret & Bulbulcus ibis & $\mathrm{R}, \mathrm{C}$ \\
\hline 17 & Little Egret & Egretta garzetta & $\mathrm{R}, \mathrm{C}$ \\
\hline 18 & Large Egret & Casmerodius albus & $\mathrm{R}, \mathrm{C}$ \\
\hline 19 & Little Heron & Butorides striatus & $\mathrm{R}, \mathrm{C}$ \\
\hline 20 & Indian Pond Heron & Ardeola grayii & $\mathrm{R}, \mathrm{C}$ \\
\hline 21 & Cinnamon Bittern & Ixobrychus cinnamomeus & $R, r$ \\
\hline \multirow[t]{2}{*}{22} & Yellow Bittern & Ixobrychus sinensis & $\mathrm{R}, \mathrm{r}$ \\
\hline & Phalacrocoracidae & & \\
\hline 23 & Indian Cormorant & Phalacrocorax fuscicollis & $\mathrm{R}, \mathrm{r}$ \\
\hline 24 & Great Cormorant & Phalacrocorax carbo & WM, C \\
\hline \multirow[t]{2}{*}{25} & Little Cormorant & Microcarbo niger & $\mathrm{R}, \mathrm{C}$ \\
\hline & Anhingidae & & \\
\hline \multirow[t]{2}{*}{26} & Oriental Darter & Anhinga melanogaster & $\begin{array}{l}\mathrm{NT}, \\
\mathrm{R}, \mathrm{r}\end{array}$ \\
\hline & Accipitridae & & \\
\hline 27 & Crested Serpent Eagle & Spilornis cheela & $\mathrm{R}, \mathrm{C}$ \\
\hline 28 & Eurasian Sparrowhawk & Accipiter nisus & $\mathrm{R}, \mathrm{C}$ \\
\hline 29 & Crested Goshawk & Accipiter trivirgatus & $\mathrm{R}, \mathrm{r}$ \\
\hline 30 & Pied Harrier & Circus melanoleucos & WM, r \\
\hline 31 & Black Kite & Milvus migrans & $\mathrm{R}, \mathrm{r}$ \\
\hline 32 & Shikra & Accipiter badius & $\mathrm{R}, \mathrm{C}$ \\
\hline 33 & Long-billed Vulture & Gyps indicus & $\begin{array}{l}\mathrm{R}, \mathrm{r} \\
(\mathrm{CR})\end{array}$ \\
\hline
\end{tabular}

\begin{tabular}{|c|c|c|c|}
\hline & Common name & Scientific name & Status \\
\hline 34 & $\begin{array}{l}\text { Indian White-backed } \\
\text { Vulture }\end{array}$ & Gyps bengalensis & $\begin{array}{l}\mathrm{CR}, \\
\mathrm{R}, \mathrm{r}\end{array}$ \\
\hline \multirow[t]{2}{*}{35} & $\begin{array}{l}\text { Greater Grey-headed } \\
\text { Fish-Eagle }\end{array}$ & Ichthyophaga ichthyaetus & $\begin{array}{l}\text { NT, } \\
\text { R, r }\end{array}$ \\
\hline & Falconidae & & \\
\hline \multirow[t]{2}{*}{36} & Common Kestrel & Falco tinnunculus & WM, C \\
\hline & Rallidae & & \\
\hline 37 & $\begin{array}{l}\text { White-breasted } \\
\text { Waterhen }\end{array}$ & Amaurornis phoenicurus & $\mathrm{R}, \mathrm{C}$ \\
\hline 38 & Water Cock & Gallicrex cinerea & $\mathrm{R}, \mathrm{r}$ \\
\hline 39 & Purple Moorhen & Porphyrio porphyrio & WM, C \\
\hline \multirow[t]{2}{*}{40} & Common Moorhen & Gallinula chloropus & WM, C \\
\hline & Charadridae & & \\
\hline 41 & Little-ring Plover & Charadrius dubius & $\mathrm{R}, \mathrm{C}$ \\
\hline 42 & Lesser Sand Plover & Charadrius mongolus & WM, C \\
\hline 43 & Little Stint & Calidris minuta & WM, C \\
\hline 44 & Red-wattled Lapwing & Vanellus indicus & $\mathrm{R}, \mathrm{C}$ \\
\hline \multirow[t]{2}{*}{45} & River Lapwing & Vanellus duvaucelii & $\mathrm{R}, \mathrm{C}$ \\
\hline & Scolopacidae & & \\
\hline 46 & Common Snipe & Gallinago gallinago & WM, r \\
\hline 47 & Pintail Snipe & Gallinago stenura & WM, C \\
\hline 48 & Common Greenshank & Tringa nebularia & WM, C \\
\hline \multirow[t]{2}{*}{49} & Common Sandpiper & Actitis hypoleucos & WM, C \\
\hline & Laridae & & \\
\hline \multirow[t]{2}{*}{50} & River Tern & Sterna aurantia & $\mathrm{R}, \mathrm{C}$ \\
\hline & Columbidae & & \\
\hline 51 & $\begin{array}{l}\text { Pompadour Green } \\
\text { Pigeon }\end{array}$ & Treron pompadoura & $\mathrm{R}, \mathrm{C}$ \\
\hline 52 & $\begin{array}{l}\text { Yellow-footed Green } \\
\text { Pigeon }\end{array}$ & Treron phoenicoptera & $\mathrm{R}, \mathrm{C}$ \\
\hline 53 & $\begin{array}{l}\text { Thick-billed Green } \\
\text { Pigeon }\end{array}$ & Treron curvirostra & $\mathrm{R}, \mathrm{r}$ \\
\hline 54 & $\begin{array}{l}\text { Pin-tailed Green } \\
\text { Pigeon }\end{array}$ & Treron apicauda & $\mathrm{R}, \mathrm{C}$ \\
\hline 55 & $\begin{array}{l}\text { Wedge-tailed Green } \\
\text { Pigeon }\end{array}$ & Treron sphenura & $\mathrm{R}, \mathrm{C}$ \\
\hline 56 & Green Imperial Pigeon & Ducula aenea & $\mathrm{R}, \mathrm{C}$ \\
\hline 57 & $\begin{array}{l}\text { Mountain Imperial } \\
\text { Pigeon }\end{array}$ & Ducula badia & $\mathrm{R}, \mathrm{C}$ \\
\hline 58 & Purple Wood Pigeon & Columba punicea & $\begin{array}{l}\text { R, r, } \\
\text { VU }\end{array}$ \\
\hline 59 & Ashy Wood Pigeon & Columba pulchricollis & $R, r$ \\
\hline 60 & Spotted Dove & Streptopelia chinensis & $\mathrm{R}, \mathrm{C}$ \\
\hline 61 & Oriental Turtle Dove & Streptopelia orientalis & $\mathrm{R}, \mathrm{r}$ \\
\hline 62 & Red Collared Dove & $\begin{array}{l}\text { Streptopelia } \\
\text { tranquebarica }\end{array}$ & $\mathrm{R}, \mathrm{r}$ \\
\hline 63 & Emerald Dove & Chalcophaps indica & $R, r$ \\
\hline 64 & Eurasian Collard Dove & Streptopelia decaocto & $R, r$ \\
\hline 65 & Barred Cuckoo Dove & Macropygia unchall & $\mathrm{R}, \mathrm{r}$ \\
\hline
\end{tabular}




\begin{tabular}{|c|c|c|c|}
\hline & Common name & Scientific name & Status \\
\hline & Psittacidae & & \\
\hline 66 & Rose-ringed Parakeet & Psittacula krameri & $\mathrm{R}, \mathrm{C}$ \\
\hline 67 & Alexandrine Parakeet & Psittacula eupatria & $\mathrm{R}, \mathrm{C}$ \\
\hline 68 & Red-breasted Parakeet & Psittacula alexandri & $\mathrm{R}, \mathrm{C}$ \\
\hline \multirow[t]{2}{*}{69} & $\begin{array}{l}\text { Blossom-headed } \\
\text { Parakeet }\end{array}$ & Psittacula roseata & $R, r$ \\
\hline & Cuculidae & & \\
\hline 70 & Drongo Cuckoo & Surniculus lugubris & $S M, r$ \\
\hline 71 & Large Hawk Cuckoo & $\begin{array}{l}\text { Heirococcyx } \\
\text { sparverioides }\end{array}$ & $R, r$ \\
\hline 72 & Common Hawk Cuckoo & Heirococcyx varius & $R, r$ \\
\hline 73 & Indian Cuckoo & Cuculus micropterus & $\mathrm{R}, \mathrm{C}$ \\
\hline 74 & $\begin{array}{l}\text { Rufous-bellied Plaintive } \\
\text { Cuckoo }\end{array}$ & Cacomantis merulinus & $\mathrm{R}, \mathrm{r}$ \\
\hline 75 & Pied Crested Cuckoo & Clamator jacobinus & $S M, r$ \\
\hline 76 & $\begin{array}{l}\text { Red-winged Crested } \\
\text { Cuckoo }\end{array}$ & Clamator coromandus & $R, r$ \\
\hline 77 & Asian Koel & Eudynamys scolopacea & $\mathrm{R}, \mathrm{C}$ \\
\hline 78 & Green-billed Malkoha & Phaenicophaeus tristis & $\mathrm{R}, \mathrm{C}$ \\
\hline 79 & Lesser Coucal & Centropus bengalesis & $\mathrm{R}, \mathrm{C}$ \\
\hline \multirow[t]{2}{*}{80} & Greater Coucal & Centropus sinensis & $\mathrm{R}, \mathrm{C}$ \\
\hline & Strigidae & & \\
\hline 81 & Spotted Owlet & Athene brama & $\mathrm{R}, \mathrm{r}$ \\
\hline 82 & Collared Owlet & Glaucidium brodiei & $R, r$ \\
\hline 83 & Asian Barred Owlet & Glaucidium cuculoides & $R, r$ \\
\hline 84 & Brown Fish Owl & Ketupa zeylonensis & $\mathrm{R}, \mathrm{r}$ \\
\hline \multirow[t]{2}{*}{85} & Spotted Scops Owl & Otus spilocephalus & $R, r$ \\
\hline & Caprimulgidae & & \\
\hline \multirow[t]{2}{*}{86} & Grey Nightjar & Caprimulgus indicus & $\mathrm{R}, \mathrm{r}$ \\
\hline & Apodidae & & \\
\hline 87 & Asian Palm Swift & Cypsiurus balasiensis & $\mathrm{R}, \mathrm{C}$ \\
\hline 88 & Himalayan Swiftlet & Collocalia fuciphaga & $\mathrm{R}, \mathrm{r}$ \\
\hline \multirow[t]{2}{*}{89} & House Swift & Apus affinis & $\mathrm{R}, \mathrm{C}$ \\
\hline & Trogonidae & & \\
\hline \multirow[t]{2}{*}{90} & Red-headed Trogon & $\begin{array}{l}\text { Herpactes } \\
\text { erythrocephalus }\end{array}$ & $\mathrm{R}, \mathrm{C}$ \\
\hline & Coraciidae & & \\
\hline 91 & Indian Roller & Coracias benghalensis & $\mathrm{R}, \mathrm{C}$ \\
\hline \multirow[t]{2}{*}{92} & $\begin{array}{l}\text { Oriental Broad-billed } \\
\text { Roller }\end{array}$ & Eurystomus orientalis & $\mathrm{R}, \mathrm{C}$ \\
\hline & Alcedinidae & & \\
\hline 93 & $\begin{array}{l}\text { White-breasted } \\
\text { Kingfisher }\end{array}$ & Halycyon smyrensis & $\mathrm{R}, \mathrm{C}$ \\
\hline 94 & Common Kingfisher & Alcedo atthis & $\mathrm{R}, \mathrm{C}$ \\
\hline 95 & Crested Kingfisher & Magaceryle lugubris & $\mathrm{R}, \mathrm{C}$ \\
\hline 96 & Ruddy Kingfisher & Halycyon coromanda & $\mathrm{R}, \mathrm{r}$ \\
\hline 97 & $\begin{array}{l}\text { Oriental Dwarf } \\
\text { Kingfisher }\end{array}$ & Ceyx erithacus & $\mathrm{R}, \mathrm{r}$ \\
\hline 98 & Pied Kingfisher & Ceryle rudis & $\mathrm{R}, \mathrm{C}$ \\
\hline 99 & Blue-eared Kingfisher & Alcedo meninting & $R, r$ \\
\hline
\end{tabular}

\begin{tabular}{|c|c|c|c|}
\hline & Common name & Scientific name & Status \\
\hline \multirow[t]{2}{*}{100} & Stork-billed Kingfisher & Halcyon capansis & $\mathrm{R}, \mathrm{r}$ \\
\hline & Meropidae & & \\
\hline 101 & Green Bee-eater & Merops orientalis & $\mathrm{R}, \mathrm{C}$ \\
\hline \multirow[t]{2}{*}{102} & $\begin{array}{l}\text { Blue-bearded Bee- } \\
\text { eater }\end{array}$ & Nyctyornis athertoni & $\mathrm{R}, \mathrm{r}$ \\
\hline & Upupidae & & \\
\hline \multirow[t]{2}{*}{103} & Hoopoe & Upupa epops & $\mathrm{R}, \mathrm{C}$ \\
\hline & Bucerotidae & & \\
\hline 104 & Oriental Pied Hornbill & Anthracoceros albrostris & $\mathrm{R}, \mathrm{C}$ \\
\hline 105 & Brown Hornbill & Anorrhinus tickelli & $\begin{array}{l}\mathrm{R}, \mathrm{r}, \\
\mathrm{En} \\
(\mathrm{NT})\end{array}$ \\
\hline 106 & Wreathed Hornbill & Aceros undulatus & $\mathrm{R}, \mathrm{C}$ \\
\hline \multirow[t]{2}{*}{107} & Great Pied Hornbill & Buceros bicornis & $\begin{array}{l}\mathrm{R}, \mathrm{r} \\
\text { (NT) }\end{array}$ \\
\hline & Megalaimidae & & \\
\hline 108 & Coppersmith Barbet & $\begin{array}{l}\text { Megalaima } \\
\text { haemocephala }\end{array}$ & $\mathrm{R}, \mathrm{C}$ \\
\hline 109 & Blue-throated Barbet & Megalaima asiatica & $\mathrm{R}, \mathrm{C}$ \\
\hline 110 & Lineated Barbet & Megalaima lineata & $\mathrm{R}, \mathrm{C}$ \\
\hline 111 & Great Barbet & Megalaima virens & $R, r$ \\
\hline \multirow[t]{2}{*}{112} & Golden-throated Barbet & Megalaima franklinii & $R, r$ \\
\hline & Picidae & & \\
\hline 113 & $\begin{array}{l}\text { Fulvous-breasted } \\
\text { Woodpecker }\end{array}$ & Dendrocopos macei & $R, r$ \\
\hline 114 & $\begin{array}{l}\text { Grey-headed } \\
\text { Woodpecker }\end{array}$ & Picus canus & $R, r$ \\
\hline 115 & $\begin{array}{l}\text { Greater Yellownape } \\
\text { Woodpecker }\end{array}$ & Picus flavinucha & $\mathrm{R}, \mathrm{C}$ \\
\hline 116 & $\begin{array}{l}\text { Lesser Yelloenape } \\
\text { Woodpecker }\end{array}$ & Picus chlorolophus & $\mathrm{R}, \mathrm{C}$ \\
\hline 117 & Rufous Woodpecker & Celeus brachyurus & $\mathrm{R}, \mathrm{C}$ \\
\hline 118 & Bay Woodpecker & Blythipicus pyrrhotis & $\mathrm{R}, \mathrm{C}$ \\
\hline 119 & Himalayan Flameback & Dinopium shorii & $\mathrm{R}, \mathrm{C}$ \\
\hline 120 & Greater Flameback & Chrysocolaptes lucidus & $R, r$ \\
\hline 121 & $\begin{array}{l}\text { Pale-headed } \\
\text { Woodpecker }\end{array}$ & Gecinulus grantia & $R, r$ \\
\hline 122 & $\begin{array}{l}\text { Crimson-breasted } \\
\text { Woodpecker }\end{array}$ & Dendrocopos cathpharius & $R, r$ \\
\hline 123 & White-browed Piculet & Sasia ochracea & $R, r$ \\
\hline \multirow[t]{2}{*}{124} & Speckled Piculet & Picumnus innominatus & $R, r$ \\
\hline & Pittidae & & \\
\hline 125 & Blue Pitta & Pita cyanea & $R, r$ \\
\hline \multirow[t]{2}{*}{126} & Blue-naped Pitta & Pitta nipalensis & $R, r$ \\
\hline & Aegithinidae & & \\
\hline \multirow[t]{2}{*}{127} & Common Lora & Aegithina tiphia & $R, r$ \\
\hline & Campephagidae & & \\
\hline 128 & Rosy Minivet & Pericrocotus roseus & WM, $r$ \\
\hline 129 & Scarlet Minivet & Pericrocotus flammeus & $\mathrm{R}, \mathrm{C}$ \\
\hline 130 & Grey-chinned Minivet & Pericrocotus solaris & $\mathrm{R}, \mathrm{C}$ \\
\hline 131 & Long-tailed Minivet & Pericrocotus ethologus & $\mathrm{R}, \mathrm{C}$ \\
\hline
\end{tabular}




\begin{tabular}{|c|c|c|c|}
\hline & Common name & Scientific name & Status \\
\hline 132 & Large Cuckoo-shrike & Coracina macei & $\mathrm{R}, \mathrm{C}$ \\
\hline 133 & $\begin{array}{l}\text { Black-winged Cuckoo- } \\
\text { shrike }\end{array}$ & Coracina melaschistos & $\mathrm{R}, \mathrm{C}$ \\
\hline 134 & Large Wood-shrike & Tephrodornis gularis & $\mathrm{R}, \mathrm{C}$ \\
\hline \multirow[t]{2}{*}{135} & Pied Flycatcher-shrike & Hemipus picatus & $\mathrm{R}, \mathrm{r}$ \\
\hline & Lanidae & & \\
\hline 136 & Brown Shrike & Lanius cristatus & WM, $r$ \\
\hline \multirow[t]{2}{*}{137} & Grey-backed Shrike & Lanius tephronotus & $\mathrm{R}, \mathrm{r}$ \\
\hline & Monarchidae & & \\
\hline \multirow[t]{2}{*}{138} & $\begin{array}{l}\text { Asian Paradise } \\
\text { Flycatcher }\end{array}$ & Terpsiphone paradisi & SM, r \\
\hline & Motacillidae & & \\
\hline 139 & White Wagtail & Motacilla alba & WM, C \\
\hline 140 & Grey Wagtail & Motacilla cinerea & WM, $r$ \\
\hline 141 & Citrine Wagtail & Motacilla citreola & WM, C \\
\hline \multirow[t]{2}{*}{142} & Paddyfield Pipit & Anthus rufulus & $\mathrm{R}, \mathrm{C}$ \\
\hline & Paridae & & \\
\hline 143 & Great Tit & Parus major & $\mathrm{R}, \mathrm{C}$ \\
\hline 144 & Sultan Tit & Melanochlora sultanea & $\mathrm{R}, \mathrm{C}$ \\
\hline \multirow[t]{2}{*}{145} & Green-backed Tit & Parus monticolus & $R, r$ \\
\hline & Timaliidae & & \\
\hline 146 & Striated Marsh-Warbler & Megalurus palustris & $\mathrm{R}, \mathrm{C}$ \\
\hline 147 & Abott's babbler & Malacocinda abbotti & $\mathrm{R}, \mathrm{C}$ \\
\hline 148 & Rufous-capped Babbler & Stachyris ruficeps & $\mathrm{R}, \mathrm{C}$ \\
\hline 149 & Golden Babbler & Stachyris chrysaea & $\mathrm{R}, \mathrm{C}$ \\
\hline 150 & Spot-throated Babbler & Pnoepyga albiventre & $\mathrm{R}, \mathrm{C}$ \\
\hline 151 & Pygmy Wren Babbler & Pnoepyga pusilla & $\mathrm{R}, \mathrm{C}$ \\
\hline 152 & $\begin{array}{l}\text { Chestnut-capped } \\
\text { Babbler }\end{array}$ & Timalia pileata & $\mathrm{R}, \mathrm{C}$ \\
\hline 153 & $\begin{array}{l}\text { White-browed Scimitar } \\
\text { Babbler }\end{array}$ & Pomatorhinus schisticeps & $\mathrm{R}, \mathrm{C}$ \\
\hline 154 & $\begin{array}{l}\text { Streak-breasted } \\
\text { Scimitar Babbler }\end{array}$ & Pomatorhinus ruficollis & $\mathrm{R}, \mathrm{C}$ \\
\hline 155 & $\begin{array}{l}\text { Wedge-billed Wren } \\
\text { Babbler }\end{array}$ & Sphenocichla humei & $\begin{array}{l}\mathrm{R}, \mathrm{r} \\
\mathrm{En} \\
(\mathrm{NT})\end{array}$ \\
\hline 156 & Marsh Babbler & Pellorneum palustre & $\begin{array}{l}\mathrm{R}, \mathrm{r} \\
\mathrm{En} \\
(\mathrm{NT})\end{array}$ \\
\hline 157 & Striped Tit Babbler & Macronous gularis & $\mathrm{R}, \mathrm{C}$ \\
\hline 158 & Yellow-eyed Babbler & Chrysomma sinense & $\mathrm{R}, \mathrm{C}$ \\
\hline 159 & Striated Babbler & Turdoides earlei & $\mathrm{R}, \mathrm{C}$ \\
\hline 160 & Slender-billed babbler & Turdoides longitostris & $\begin{array}{l}\mathrm{R}, \mathrm{r} \\
(\mathrm{VU})\end{array}$ \\
\hline 161 & Jungle Babbler & Turdoides striatus & $\mathrm{R}, \mathrm{C}$ \\
\hline 162 & Silver-eared Mesia & Leiothrix argentauris & $\mathrm{R}, \mathrm{C}$ \\
\hline 163 & $\begin{array}{l}\text { White-crested } \\
\text { Laughing Thrush }\end{array}$ & Garrulax leucolophus & $R, r$ \\
\hline 164 & $\begin{array}{l}\text { White-throated } \\
\text { Laughing thrush }\end{array}$ & Garrulax albogularis & $\mathrm{R}, \mathrm{r}$ \\
\hline
\end{tabular}

\begin{tabular}{|c|c|c|c|}
\hline & Common name & Scientific name & Status \\
\hline 165 & $\begin{array}{l}\text { Greater-necklace } \\
\text { Laughing Thrush }\end{array}$ & Garrulax pectoralis & $\mathrm{R}, \mathrm{C}$ \\
\hline 166 & $\begin{array}{l}\text { Lesser-necklace } \\
\text { Laughing Thrush }\end{array}$ & Garrulax monileger & $\mathrm{R}, \mathrm{C}$ \\
\hline 167 & $\begin{array}{l}\text { Striated Laughing } \\
\text { Thrush }\end{array}$ & Garrulax striatus & $\mathrm{R}, \mathrm{C}$ \\
\hline 168 & $\begin{array}{l}\text { Rufous-vented } \\
\text { Laughing Thrush }\end{array}$ & Garrulax gularis & $\mathrm{R}, \mathrm{C}$ \\
\hline 169 & $\begin{array}{l}\text { Rufous-necked } \\
\text { Laughing Thrush }\end{array}$ & Garrulax ruficollis & $R, r$ \\
\hline 170 & Blue Rock-Thrush & Monticola solitarius & WM, C \\
\hline 171 & Red Faced Liocichla & Liocichla phoenicea & $\mathrm{R}, \mathrm{C}$ \\
\hline 172 & White-naped Yuhina & Yuhina bakeri & $\begin{array}{l}\mathrm{R}, \mathrm{r} \\
\mathrm{En}\end{array}$ \\
\hline 173 & White-bellied Yuhina & Yuhina zantholeuca & $\mathrm{R}, \mathrm{r}$ \\
\hline 174 & Black-chinned Yuhina & Yuhina nigrimenta & $\mathrm{R}, \mathrm{r}$ \\
\hline 175 & Whiskered Yuhina & Yuhina flavicollis & $\mathrm{R}, \mathrm{C}$ \\
\hline 176 & Nepal Fulvetta & Alcippe nipalensis & $\mathrm{R}, \mathrm{C}$ \\
\hline 177 & Long-tailed Sibia & Heterophasia picaodes & $\mathrm{R}, \mathrm{C}$ \\
\hline 178 & Beautiful Sibia & Heterophasia pulchella & $\begin{array}{l}\mathrm{R}, \mathrm{r} \\
\mathrm{En}\end{array}$ \\
\hline 179 & Cutia & Cutia nipalensis & $\mathrm{R}, \mathrm{r}$ \\
\hline 180 & Common Tailorbird & Orthotomus sutorius & $\mathrm{R}, \mathrm{C}$ \\
\hline \multirow[t]{2}{*}{181} & Mountain Tailorbird & Orthotomus cuculatus & $\mathrm{R}, \mathrm{C}$ \\
\hline & Phyllocopidae & & \\
\hline 182 & Grey-cheeked Warbler & Seicercus poliogenys & $\mathrm{R}, \mathrm{C}$ \\
\hline 183 & Grey-hooded Warbler & Seicercus xanthoschistos & $\mathrm{R}, \mathrm{C}$ \\
\hline \multirow[t]{2}{*}{184} & Greenish Warbler & Phylloscopus trochiloides & WM, $r$ \\
\hline & Cettidae & & \\
\hline 185 & Black-faced Warbler & Abroscopus schisticeps & $\mathrm{R}, \mathrm{C}$ \\
\hline 186 & Rufous-faced Warbler & Abroscopus albogularis & $\mathrm{R}, \mathrm{C}$ \\
\hline 187 & $\begin{array}{l}\text { Grey-sided Bush- } \\
\text { warbler }\end{array}$ & Cettia brunnifrons & $\mathrm{R}, \mathrm{C}$ \\
\hline \multirow[t]{2}{*}{188} & Aberrant-bush warbler & Cettia flavolivacea & $\mathrm{R}, \mathrm{r}$ \\
\hline & Muscicapidae & & \\
\hline 189 & $\begin{array}{l}\text { Grey-headed Canary- } \\
\text { flycatcher }\end{array}$ & Culicicapa ceylonensis & $R, r$ \\
\hline 190 & Pale Blue-flycatcher & Muscicapa unicolor & $\mathrm{R}, \mathrm{r}$ \\
\hline 191 & Little Pied-flycatcher & Ficedula westermanni & $R, r$ \\
\hline 192 & $\begin{array}{l}\text { White Gorgeted- } \\
\text { flycatcher }\end{array}$ & Ficedula monileger & $\mathrm{R}, \mathrm{r}$ \\
\hline 193 & Sapphire Flycatcher & Ficedula sapphira & $R, r$ \\
\hline 194 & $\begin{array}{l}\text { Rufous Gorgeted- } \\
\text { flycatcher }\end{array}$ & Ficedula strophiata & $R, r$ \\
\hline 195 & $\begin{array}{l}\text { Snowy Browed- } \\
\text { flycatcher }\end{array}$ & Ficedula hyperythra & $R, r$ \\
\hline 196 & Pygmy Blue-flycatcher & Muscicapella hodgsoni & $\mathrm{R}, \mathrm{r}$ \\
\hline 197 & Magpie Robin & Copsychus saularis & $\mathrm{R}, \mathrm{C}$ \\
\hline 198 & Common Stonechat & Saxicola torquata & WM, r \\
\hline 199 & Grey Bushchat & Saxicola ferrea & $\mathrm{R}, \mathrm{C}$ \\
\hline 200 & White-rumped Shama & Copsychus malabaricus & $R, r$ \\
\hline
\end{tabular}




\begin{tabular}{|c|c|c|c|}
\hline & Common name & Scientific name & Status \\
\hline 201 & $\begin{array}{l}\text { Rufous-breasted Bush } \\
\text { Robin }\end{array}$ & Tarsiger indicus & $R, r$ \\
\hline 202 & White-crowned Forktail & Enicurus leschenaulti & $\mathrm{R}, \mathrm{C}$ \\
\hline 203 & Black-backed Forktail & Enicurus immaculatus & $\mathrm{R}, \mathrm{C}$ \\
\hline 204 & Slaty-backed Forktail & Enicurus schistaceus & $\mathrm{R}, \mathrm{C}$ \\
\hline 205 & Small Niltava & Niltava macgrigoriae & $\mathrm{R}, \mathrm{C}$ \\
\hline 206 & Green Cochoa & Cochoa viridis & $\mathrm{SM}, \mathrm{r}$ \\
\hline 207 & $\begin{array}{l}\text { White-capped Water- } \\
\text { redstart }\end{array}$ & $\begin{array}{l}\text { Chaimarrornis } \\
\text { leucocephalus }\end{array}$ & $R, r$ \\
\hline 208 & Daurian Redstart & Phoenicurus auroreus & WM, $r$ \\
\hline \multirow[t]{2}{*}{209} & $\begin{array}{l}\text { Plumbeous Water- } \\
\text { redstart }\end{array}$ & Rhyacornis fuliginosus & $\mathrm{R}, \mathrm{C}$ \\
\hline & Cisticolidae & & \\
\hline 210 & Grey-breasted Prinia & Prinia hodgsonii & $\mathrm{R}, \mathrm{C}$ \\
\hline 211 & Striated Prinia & Prinia criniger & $\mathrm{R}, \mathrm{C}$ \\
\hline \multirow[t]{2}{*}{212} & Beavan's Prinia & Prinia rufescens & $\mathrm{R}, \mathrm{C}$ \\
\hline & Turdidae & & \\
\hline 213 & Blue Whistling Thrush & Myophonus caeruleus & $\mathrm{R}, \mathrm{C}$ \\
\hline 214 & Orange headed Thrush & Zoothera citrina (SV) & SM, r \\
\hline \multirow[t]{2}{*}{215} & Scaly Thrush & Zoothera dauma & WM, C \\
\hline & Chloropseidae & & \\
\hline 216 & Blue-winged Leafbird & $\begin{array}{l}\text { Chloropsis } \\
\text { cochinchinensis }\end{array}$ & $\mathrm{R}, \mathrm{C}$ \\
\hline 217 & $\begin{array}{l}\text { Golden-fronted } \\
\text { Leafbird }\end{array}$ & Chloropsis aurifrons & $\mathrm{R}, \mathrm{C}$ \\
\hline \multirow[t]{2}{*}{218} & Asian Fairy-bluebird & Irena puella & $\mathrm{R}, \mathrm{C}$ \\
\hline & Pycnonotidae & & \\
\hline 219 & Red-vented Bulbul & Pycnonotus cafer & $\mathrm{R}, \mathrm{C}$ \\
\hline 220 & Red-whiskered Bulbul & Pycnonotus jocosus & $\mathrm{R}, \mathrm{C}$ \\
\hline 221 & White-throated Bulbul & Alophoixus flaveolus & $\mathrm{R}, \mathrm{C}$ \\
\hline 222 & Ashy Bulbul & Hemixos flava & $\mathrm{R}, \mathrm{C}$ \\
\hline 223 & Himalayan Bulbul & Pycnonotus leucogenys & $\mathrm{R}, \mathrm{r}$ \\
\hline 224 & Striated Bulbul & Pycnonotus striatus & $\mathrm{R}, \mathrm{C}$ \\
\hline 225 & Black Bulbul & $\begin{array}{l}\text { Hypsipetes } \\
\text { leucocephalus }\end{array}$ & $\mathrm{R}, \mathrm{r}$ \\
\hline 226 & Black-crested Bulbul & Pycnonotus melanicterus & $\mathrm{R}, \mathrm{C}$ \\
\hline 227 & Mountain Bulbul & Hypsipetes mcclellandii & $\mathrm{R}, \mathrm{C}$ \\
\hline \multirow[t]{2}{*}{228} & Crested Finchbill & Spizixos canifrons & $\mathrm{R}, \mathrm{C}$ \\
\hline & Sittidae & & \\
\hline 229 & Velvet-fronted Nuthatch & Sitta frontalis & $\mathrm{R}, \mathrm{C}$ \\
\hline \multirow[t]{2}{*}{230} & $\begin{array}{l}\text { Chestnut-bellied } \\
\text { Nuthatch }\end{array}$ & Sitta castanea & $\mathrm{R}, \mathrm{C}$ \\
\hline & Saturnidae & & \\
\hline 231 & Common Myna & Acridotheres tristis & $\mathrm{R}, \mathrm{C}$ \\
\hline 232 & Pied Myna & Sturnus contra & $\mathrm{R}, \mathrm{C}$ \\
\hline 233 & Jungle Myna & Acridotheres fuscus & $\mathrm{R}, \mathrm{C}$ \\
\hline 234 & Grey-headed Myna & Sturnus malabaricus & $\mathrm{R}, \mathrm{C}$ \\
\hline \multirow[t]{2}{*}{235} & Hill Myna & Gracula religiosa & $R, r$ \\
\hline & Oriolidae & & \\
\hline 236 & Black-headed oriole & Oriolus xanthornus & $\mathrm{R}, \mathrm{C}$ \\
\hline
\end{tabular}

\begin{tabular}{|c|c|c|c|}
\hline & Common name & Scientific name & Status \\
\hline \multirow[t]{2}{*}{237} & Maroon Oriole & Oriolus traillii & $\mathrm{R}, \mathrm{C}$ \\
\hline & Dicruridae & & \\
\hline 238 & Black Drongo & Dicrurus macrocercus & $\mathrm{R}, \mathrm{r}$ \\
\hline 239 & Bronze Drongo & Dicrurus aeneus & $\mathrm{R}, \mathrm{C}$ \\
\hline 240 & Spangled Drongo & Dicrurus hottentottus & $\mathrm{R}, \mathrm{C}$ \\
\hline 241 & Ashy Drongo & Dicrurus leucophaeus & WM, r \\
\hline 242 & $\begin{array}{l}\text { Lesser Racket-tailed } \\
\text { Drongo }\end{array}$ & Dicrurus remifer & $R, r$ \\
\hline \multirow[t]{2}{*}{243} & $\begin{array}{l}\text { Greater Racket-tailed } \\
\text { Drongo }\end{array}$ & Dicrurus paradiseus & $R, r$ \\
\hline & Corvidae & & \\
\hline 244 & Rufous Treepie & Dendrocitta vagabunda & $\mathrm{R}, \mathrm{r}$ \\
\hline 245 & Grey Treepie & Dendrocitta formosae & $\mathrm{R}, \mathrm{r}$ \\
\hline 246 & Collared Treepie & Dendrocitta frontalis & $\mathrm{R}, \mathrm{r}$ \\
\hline 247 & $\begin{array}{l}\text { Common Green } \\
\text { Magpie }\end{array}$ & Cissa chinensis & $R, r$ \\
\hline 248 & Jungle Crow & Corvus macrorhynchus & $\mathrm{R}, \mathrm{C}$ \\
\hline 249 & Common Crow & Corvus splendens & $\mathrm{R}, \mathrm{C}$ \\
\hline 250 & White-throated Fantail & Rhipidura albicollis & $\mathrm{R}, \mathrm{r}$ \\
\hline \multirow[t]{2}{*}{251} & Eurasian Jay & Garrulus glandarius & $R, r$ \\
\hline & Passeridae & & \\
\hline \multirow[t]{2}{*}{252} & House Sparrow & Passer domesticus & $\mathrm{R}, \mathrm{C}$ \\
\hline & Ploceidae & & \\
\hline \multirow[t]{2}{*}{253} & Baya Weaver & Ploceus philippinus & $\mathrm{R}, \mathrm{r}$ \\
\hline & Zosteropidae & & \\
\hline \multirow[t]{2}{*}{254} & Oriental white-eye & Zosterops palpebrosus & $\mathrm{R}, \mathrm{C}$ \\
\hline & Estrildidae & & \\
\hline 255 & Scaly-breasted Munia & Lonchura punctulata & $\mathrm{R}, \mathrm{C}$ \\
\hline 256 & White-rumped Munia & Lonchura striata & $\mathrm{R}, \mathrm{C}$ \\
\hline \multirow[t]{2}{*}{257} & Black-headed Munia & Lonchura malacca & $\mathrm{R}, \mathrm{C}$ \\
\hline & Tichodromidae & & \\
\hline \multirow[t]{2}{*}{258} & Wallcreeper & Tichodroma muraria & W, V \\
\hline & Dicacidae & & \\
\hline 259 & $\begin{array}{l}\text { Fire-breasted } \\
\text { Flowerpecker }\end{array}$ & Dicaeum ignipectus & $\mathrm{R}, \mathrm{C}$ \\
\hline \multirow[t]{2}{*}{260} & $\begin{array}{l}\text { Scarlet-backed } \\
\text { Flowerpecker }\end{array}$ & Dicaeum cruentatum & $\mathrm{R}, \mathrm{C}$ \\
\hline & Nectariniidae & & \\
\hline 261 & Purple-rumped sunbird & Nectarinia zeylonica & $\mathrm{R}, \mathrm{C}$ \\
\hline 262 & Purple Sunbird & Nectarinia asiatica & $R, r$ \\
\hline 263 & Crimson sunbird & Aethopyga siparaja & $\mathrm{R}, \mathrm{C}$ \\
\hline 264 & Green-tailed Sunbird & Aethopyga nipalensis & $\mathrm{R}, \mathrm{C}$ \\
\hline 265 & Ruby-cheeked Sunbird & Anthreptes singalensis & $\mathrm{R}, \mathrm{C}$ \\
\hline 266 & Fire-tailed Sunbird & Aethopyga ignicauda & $R, r$ \\
\hline 267 & Streaked Spiderhunter & Arachnothera magna & $\mathrm{R}, \mathrm{r}$ \\
\hline \multirow[t]{2}{*}{268} & Little Spiderhunter & Arachnothera longirostra & $\mathrm{R}, \mathrm{C}$ \\
\hline & Aegithalidae & & \\
\hline 269 & Black-throated Bushtit & Aegithalos concinnus & $\mathrm{R}, \mathrm{C}$ \\
\hline
\end{tabular}




\begin{tabular}{|l|l|l|l|}
\hline & Common name & Scientific name & Status \\
\hline & Hirundinidae & & \\
\hline 270 & Common Swallow & Hirundo rustica & WM, C \\
\hline
\end{tabular}

R - Resident; C - Common; r - Rare; WM - Winter Migrant; SM - Summe Migrant; V - Vagrant; EN - Endangered; En - Endemic; NT - Near Threatened; VU - Vulnerable; CR - Critically Endangered; NR - New Record.

biodiversity hotspot regions harbouring wide varieties of plants and animals inhabiting a diversified habitat mosaic (Stattersfield et al. 1998). The Jeypore Reserve Forest is one among the few remaining tropical forest patches of eastern Assam which is unique for its varied flora and fauna. The high diversity of frugivorous and insectivorous birds reveals the quality of the forest habitat. Apart from these, the study records 14 globally threatened species including two Critically Endangered species and five endemic species, which also adds to the uniqueness of this small forest patch. The site should be given first priority for conservation of rare and endemic galliforme species such as Grey Peacock Pheasant Polyplectron bicalcaratum, Kaleej Pheasant Lophura leucomelanos, White-cheeked Partridge Arborophila atrogularis and Rufousthroated Partridge Arborophila rufogularis. Beside galliformes, the forest also harbours the endangered White-winged Duck Cairina scutulata. Out of the five species of hornbills found in JRF (Choudhury 2000), four species have been recorded from the site during present survey, except the Rufous-necked Hornbill Aceros nipalensis, which has not been encountered yet. Since the reserve is continuous with the Patkai Range of Arunachal Pradesh, it provides a unique habitat not only for birds but also to many large and small carnivores, herbivores, primates and reptiles. The Insect diversity is also high including a large number of unknown arachnids for which detailed studies are required immediately. Thus the site is an ideal place for conservation of endemic and globally threatened birds and also to a large number of important flora and fauna. Due to the increase in human population the forest is presently facing disturbance in the edges which will increase in due course of time if proper conservation measures are not taken up immediately. Conservation awareness programmes among the local people is required to sensitise the people about the sustainable use of the forest resources to conserve it for future generations.

\section{REFERENCES}

Ali, S. \& S.D. Ripley (1987). A Compact Handbook of the Birds of India and Pakistan, Second Edition. Oxford University Press, Delhi, 737pp.

Bibby, C.J., N.D. Burgess \& D.A. Hall (1992). Bird Census Techniques: Academic press, London, New York, San Deigo, Boston, 248pp.

BirdLife International (2001a). Threatened Birds of Asia: The BirdLife International Red Data Book—Vol. 1. Birdlife International. Cambridge, UK, 1516pp.

BirdLife International (2001b). Threatened Birds of Asia: The BirdLife International Red Data Book-Vol. 2. Birdlife International. Cambridge, UK, 1517-3038pp.

Buckland, S.T., D.R. Anderson, K.P. Burnham \& J.L. Laake (1993). Distance Sampling: Estimating the Abundance of Biological Populations. Chapman and Hall, London, 446pp.

Champion H.G. \& S.K. Seth (1968). A Revised Survey of the Forest Types of India. The Manager of Publications, Government of India, New Delhi, 404pp.

Choudhury, A.U. (1996). Survey of the White-winged Wood Duck and Bengal Florican in Tinsukia District and adjacent areas of Assam and Arunachal Pradesh. The Rhino Foundation for Nature in North East India, Guwahati. $82 \mathrm{pp}$.

Choudhury, A.U. (1998). Status and Conservation of the White-winged Duck in eastern Assam, India. OBC Bulletin 28: 14-17.

Choudhury, A.U. (2000). The Birds of Assam. Guwahati Gibbon Books and World Wide Funds for Nature, 240pp.

Gill, F. \& M. Wright (2006). Birds of World: Recommended English Names. Princeton NJ: Princeton University Press, Version 2.2 generated on 2009-08-25.

Grimmett, R., C. Inskipp \& Inskipp (2000). The Pocket Guide to the Birds of Indian Subcontinent. Oxford University Press, Delhi, 888pp.

Islam, M.J \& A.R. Rahmani (2004). Important Bird Areas in India: Priorities Sites for Conservation. Indian Bird Conservation Network: Bombay Natural History Society and Birdlife International (UK), 1133pp.

Kakati, K. (2004). Impact of Forest Fragmentation on the Hoolock Gibbon in Assam, India. PhD Thesis. Wildlife Research Group, Department of Anatomy, Cambridge University, 230pp.

Saikia, P.K. \& M.K. Saikia (2000). Diversity of Bird Fauna in N.E. India. Journal of Assam Science Society 41(2): 379-396.

Stattersfield, A.J., M.J. Crosby, M. J. Long \& D. C. Wege (1998). Endemic Bird Areas of The World: Priorities for Biodiversity Conservation. Birdlife International. Cambridge, U.K. Conservation Series 7, 846pp.

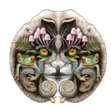

